\title{
El análisis de los elementos culturales como influencia de generación de conocimiento en las organizaciones agrícolas del Valle del Yaqui
}

\section{The analysis of the cultural elements as influence of knowledge generation in the agricultural organizations of the Yaqui Valley}

\author{
FLORES-LÓPEZ, José Guadalupe†*, OCHOA-JIMÉNEZ, Sergio y TORRES-VÁSQUEZ, María del \\ Carmen
}

Instituto Tecnológico de Sonora, Departamento de Ciencias Administrativas. Calle 5 de Febrero 818, Centro, Urb. No. 1, 85000 Cd Obregón, Son.

ID 1 ${ }^{\text {er }}$ Autor: José Guadalupe, Flores-López / ORC ID: 0000-0002-6380-513, CVU CONACYT ID: 279081

ID 1 ${ }^{\mathrm{er}}$ Coautor: Sergio, Ochoa-Jiménez / ORC ID: 0000-0003-1848-3760 CVU CONACYT ID: 286385

ID $2^{\text {do }}$ Coautor: María del Carmen, Torres-Vázquez, / ORC ID: 0000-0003-0938-4955 Research ID Thomson: X-21042018, CVU CONACYT ID: 286266

DOI: $10.35429 / J O C S .2019 .18 .6 .1 .12$

Recibido 19 de Enero, 2019; Aceptado 26 de Marzo, 2019

\begin{abstract}
Resumen
La agricultura en México es un sector relativamente pequeño ya que representó cerca de 4\% del Producto Interno Bruto (INEGI, 2009). Sin embargo, la generación de empleo es relativamente mayor, representa unos 3.3 millones de agricultores y 4.6 millones de trabajadores asalariados y familiares no remunerados. El propósito de la investigación recae en analizar la influencia de los elementos culturales en la generación de conocimiento de las organizaciones agrícolas del Valle del Yaqui para la construcción de un modelo teórico que describa una realidad. Para lo anterior, se aplicó una metodología mixta, para el estudio cuantitativo se diseñó un cuestionario de 28 ítems tipo escala Likert cinco puntos, el cual se aplicó a 90 organizaciones agrícolas del Valle del Yaqui. Para el estudio cualitativo, se diseñó una entrevista semiestructurada la cual se aplicó a siete líderes de asociaciones agrícolas y productores locales. Como resultado se obtuvo que los factores culturales influyen en la generación del conocimiento en un $10 \%$, se estima una influencia baja, y se comprueba con los resultados cualitativos, mismos que manifiestan que elementos culturales como: la tradición, las costumbres y valores son aspectos que obstaculizan la generación de un nuevo conocimiento en el Valle del Yaqui.
\end{abstract}

Cultura, Conocimiento, Agricultura

\begin{abstract}
Agriculture in Mexico is a relatively small sector since it represented around $4 \%$ of the Gross Domestic Product (INEGI, 2009). However, the generation of employment is relatively greater, representing some 3.3 million farmers and 4.6 million wage and unpaid family workers. The purpose of the research is to analyze the influence of cultural factors in the generation of knowledge of the agricultural organizations of the Yaqui Valley for the construction of a theoretical model that describes a reality. For this, a mixed methodology was applied, for the quantitative study a questionnaire of 28 Likert scale items was designed, five points, which was applied to 90 agricultural organizations of the Yaqui Valley. For the qualitative study, a semi-structured interview was designed which was applied to seven leaders of agricultural associations and local producers. As a result, it was obtained that cultural factors influence the generation of knowledge by $20 \%$, a low influence is estimated, and it is verified with qualitative results, which show that cultural elements such as tradition, customs and values are aspects that hamper the generation of new knowledge in the Yaqui Valley.
\end{abstract}

Culture, Knowledge, Agriculture

Citación: FLORES-LÓPEZ, José Guadalupe, OCHOA-JIMÉNEZ, Sergio y TORRES-VÁSQUEZ, María del Carmen. El análisis de los elementos culturales como influencia de generación de conocimiento en las organizaciones agrícolas del Valle del Yaqui. Revista de Sociología Contemporánea. 2019. 6-18: 1-12.

\footnotetext{
* Correspondencia del Autor (correo electrónico: jose.flores@itson.edu.mx)

$\uparrow$ Investigador contribuyendo como primer autor.
} 


\section{Introducción}

En México el impacto político en la agricultura se presenta cuando el estado interviene en las labores del campo y el aspecto social de la invasión de los españoles trae una pérdida de territorio e identidad en su poderío agrícola. Según datos recabados por el Instituto Nacional de Estadística, Geografía e Informática (INEGI, 2009), la agricultura en México fue un sector pequeño que representó cerca de 4\% del Producto Interno Bruto (PIB). Sin embargo, la generación de empleo fue mayor, ya que proporcionó alrededor de $13 \%$ de la fuerza de trabajo, considerando unos 3.3 millones de agricultores y 4.6 millones de trabajadores asalariados y familiares no remunerados.

La agricultura en México según Appendini, Martínez, Rendón \& Salles (1983), ha dado desde sus orígenes bastante tema de conversación y rica en historia. Se apega a lo que la misma historia del país social y revolucionaria ha acontecido, la agricultura mexicana era una de las más reconocidas en Mesoamérica, todo gracias a sus cultivos importantes del maíz, chile y frijol. México ha sido la cuna para el desarrollo e incremento de distintas plantas y frutos de cultivo, lo que ha venido a enriquecer tanto la agricultura a nivel mundial, el incremento de la alimentación; así como la base del desarrollo económico y social (FAO, 2014). En lo que refiere a extensión territorial, la SAGARPA (2007) afirma que México cuenta con un territorio nacional de 198 millones de hectáreas de las cuales 145 millones se dedican a la actividad agropecuaria, cerca de 30 millones de hectáreas son tierras de cultivo y 115 millones de agostadero. La abundancia de extensión territorial ocupada, ha generado cambios y/o desarrollos tecnológicos de gran valor.

En territorio mexicano se da una gran cantidad de cultivos, sin embargo, los 10 productos que más se producen en el campo son: caña de azúcar, maíz, plátano, sorgo, naranja, trigo, jitomate, limón, chile verde y papa. (Portal; explorando México, 2012). En el año 2009, se produjo mayormente la caña de azúcar (INEGI, 2011). En el año 2010, el vegetal que más exportó México, en base a su producción fue el trigo durum (INEGI, 2010).
Consultando el informe de la SAGARPA (2014) la producción anual agrícola del año 2013 considerando la superficie sembrada, cosechada y valor de la producción de la modalidad riego + temporal, de un total de 328 tipos de cultivos los de mayor impacto son; en cuanto a superficie sembrada el maíz grano tiene 7 487,399.02 hectáreas, los pastos $2547,929.60$ hectáreas y el sorgo grano 2 012,330.32 hectáreas y los de menor impacto son el anturios gruesa con 0.16 hectáreas, alcatraz gruesa y canola semilla con 1 hectárea.

Cabe mencionar que, en la zona norte de nuestro país, existen estados donde las áreas de cultivo se presentan mayormente para la agricultura comercial. Estos lugares se destacan por el cultivo de semillas como el trigo, maíz, garbanzo, entre otros; respecto a las hortalizas, se cuenta con papa, calabacita, espárrago, etc. El estado de Sonora es uno de los 32 estados del país que más destacan con relación a la agricultura en diversos cultivos. Tiene una extensión territorial de 184,934.0 kilómetros cuadrados los cuales representan el 9.4 por ciento del territorio del país, considerándolo como el segundo más grande en México, además es reconocido como una de las entidades con la mayor de las riquezas en agricultura, ya que según la Enciclopedia de Municipios de México con datos del año 2010 afirma que es la actividad de mayor importancia en el estado y a nivel nacional se conoce por su aportación significativa al producto interno bruto del país y es identificada con el sobrenombre de "el granero nacional" (Secretaría de Gobernación, 2010).

Los lugares que destacan dentro del estado para desarrollar la actividad agrícola son: el Valle del Mayo, Guaymas, la Costa de Hermosillo, Caborca, el Valle de San Luis Río Colorado y el Valle del Yaqui, siendo este último el más grande en superficie cultivada.

Sin embargo, el panorama a futuro por las condiciones climáticas que oscilan en el estado no son las más favorables, ya que según el instituto Nacional de Estadística y Geografía INEGI (2010) para el año 2025 habrá una disminución de la superficie cultivada particularmente en maíz, esto debido a los altos costos de los equipos de irrigación utilizados en la agricultura. 
Si bien, el estado de Sonora tiene un nivel de inversión alta en años recientes, Camiro, Altamirano y Rojas (2009), señalan que la política agrícola oficial ha orientado al Estado para la creación de la Financiera Rural como banca de desarrollo, y así encaminar sus actividades en torno al crédito agrícola como servicio fundamental del sistema, considerando que las proyecciones a futuro se invertirán en sistemas de innovación.

Según el informe Monitor Agropecuario de la SAGARPA en el año 2009 menciona que los principales cultivos que se siembran en el estado son el trigo grano, la papa, la sandía, avena y cebada entre otras. Además, varios municipios del estado han fungido como líderes productores a nivel nacional, destacando el municipio de Cajeme (ver Tabla 1).

\begin{tabular}{|l|r|r|l|l|}
\hline \multicolumn{1}{|c}{ Cultivo } & \multicolumn{1}{c}{ Superficie } & Producción & \multicolumn{1}{c|}{$\begin{array}{c}\text { Lugar } \\
\text { Nacional }\end{array}$} & \multicolumn{1}{c|}{$\begin{array}{c}\text { Municipio } \\
\text { Líder }\end{array}$} \\
\hline $\begin{array}{l}\text { Trigo } \\
\text { grano }\end{array}$ & 320,432 & $1,825,578$ & $1^{\circ}$ & Cajeme \\
\hline Papa & 9,624 & 311,590 & $2^{\circ}$ & Navojoa \\
\hline $\begin{array}{l}\text { Avena } \\
\text { forrajera }\end{array}$ & 4,323 & 126,767 & $8^{\circ}$ & Hermosillo \\
\hline $\begin{array}{l}\text { Cebada } \\
\text { forrajera } \\
\text { en verde }\end{array}$ & 3,460 & 113,253 & $2^{\circ}$ & Hermosillo \\
\hline $\begin{array}{l}\text { Maíz } \\
\text { grano }\end{array}$ & 14,349 & 92,741 & $7^{\circ}$ & Cajeme \\
\hline Sandia & 1,681 & 61,340 & $3^{\circ}$ & Empalme \\
\hline
\end{tabular}

Tabla 1 Cultivos del Estado de Sonora

Fuente: elaboración propia con base a SAGARPA (2009)

El Valle del Yaqui, comprende los municipios de Cajeme, Bacúm, San Ignacio Rio Muerto, Benito Juárez y Etchojoa; incluyen veinte mil hectáreas de las cuales $56 \%$ es tierra ejidal, el $40 \%$ de propiedad privada y el $4 \%$ de colonos. Por lo que la distribución de la superficie promedio asciende a 9 hectáreas para cada uno de los del primer grupo, 18 para el segundo y 15 para el tercero (Camiro, Altamirano \& Rojas, 2009). Según Naylor et al, (2001) es una región caracterizada por el rápido crecimiento económico y el cambio ecológico, éste recinto se compone por 235 mil hectáreas (Has) irrigadas, mismas que se encuentran entre la Sierra Madre y el Golfo de California. Del total de hectáreas localizadas que estructuran el Valle del Yaqui, cerca de 159 mil son utilizadas para la siembra de trigo, dando el 68 por ciento del total de terreno agrícola utilizado para este cultivo convirtiendo al estado como uno de los principales productores y exportadores de éste grano en el país comenta Suárez, (2012).
Con base a los datos estadísticos arrojados en 2012 del sistema de Servicios de Información Agroalimentaria y Pesquera (SIAP) el Valle del Yaqui ocupa el primer lugar a nivel estatal en superficie sembrada haciendo uso de fertilizantes químicos, sólo en el Municipio de Cajeme 108,051 mil hectáreas (19.3\% a nivel estatal) son fertilizadas mediante el mecanismo de riego. El Valle del Yaqui es admirado por su estructura y orientación, los trazos y ubicaciones de parcelas se remontan a la historia. El trazado según Cerutti y Lorenzana (2009) mencionan que pertenece a un diseño de cuadrículas dependiente de dos ejes perpendiculares: uno orientado de norte a sur y otro de este a oeste, al primero se le denominó calle Meridiano y el segundo calle base. Los ejes fueron la referencia para el trazamiento de las cuadrículas de los predios agrícolas del valle.

En datos históricos que reflejan el Valle del Yaqui, mencionan que siempre ha estado al mando de la administración de la fuente del agua el Distrito de Riego del Valle del Yaqui, sin embargo, Silos y Donald (1964) afirman que los primeros aprovechamientos del recurso vital fueron en 1980 cuando el Sr. Conant Maldonado solicitó una concesión de agua fundando la organización "Sonora-Sinaloa Irrigation C.O".

Por lo tanto, la compañía entró en problemas de liquidez y fue cuando entró "Constructora Richardson S.A" y tomó los derechos y el control del suministro de agua hasta el año de 1928.

Según el SIAP con información actualizada del año 2014, el nivel de producción del valle del Yaqui ha ido en incremento y no se han presentado pérdidas considerables en los cultivos generales del ciclo otoño-invierno y primavera-verano en consideración con el año 2013 que se perdieron cerca de 9 mil 200 has a causa de siniestros meteorológicos y plagas.

A continuación, se observa durante todo el año 2013 como el Valle del Yaqui representado por el distrito de Cajeme tiene una superficie cosechada de 269,315.69 mil has con un valor de 7,354578.58 pesos siendo el número uno en producción del estado aportando el 43 por ciento en todo el estado (ver Tabla 2). 


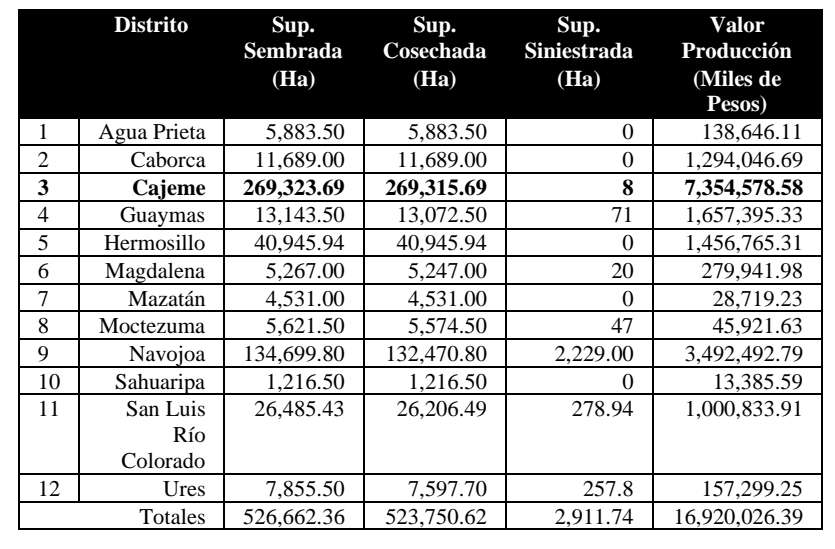

Tabla 2 Estadísticas del Valle del Yaqui en la Producción de Agrícola en el Estado

Fuente: elaboración Propia con Base a INEGI (2010)

En este lugar, se han desarrollado estudios principalmente en el uso de suelo y agua relacionado con trigo y genotipos de diferentes granos (Leal et al. 2014; Moreno et al. 2012; Ramírez, 2008; Velasco et al. 2012). Uno de los estudios consistió en el análisis de 20 genotipos de trigo harinero, para identificar mejores líneas por su rendimiento de grano, componentes del rendimiento y por su resistencia a la roya de la hoja. Son sumamente necesarios este tipo de estudio, en ese sentido, se localiza la nula participación organizacional de la investigación social en el sector.

La generación del conocimiento en el sector agrícola, es propiciada por los mismos individuos y generadas también por el sector gubernamental, es entonces que la obtención de un nuevo conocimiento puede ser generado por la perspectiva que cada persona tiene en su mente, desde sus propias ideas, factores o formas de realizar una actividad.

Considerando lo anterior, existe un estudio titulado "Implementing and maintaining a knowledge sharing culture via knowledge management teams: a shared leadership approach" elaborado por Taylor (2013) el cual propone una cultura de intercambio de conocimientos, la conducción y sostener el cambio a través del programa de gestión del conocimiento estratégico e iniciativa de proyectos enfocados en la generación de conocimientos. Se destaca también que las ideas de los líderes con conocimiento ya heredado no dejan entrar a las ideas nuevas generadas por los líderes abiertos a nuevos cambios.
De igual forma, es apropiado mencionar el siguiente estudio de investigación "Factors affecting establishment of an institutional knowledge management culture - a study of organizational visión" un caso organizacional en el cual se pretende identificar los posibles factores que pueden intervenir al querer establecer una gestión de conocimiento institucional.

El estudio fue elaborado a varias organizaciones Rumanas por Pugnaa \& Boldeanu (2014) mismo que trae consigo una metodología cualitativa en la cual se consideró una entrevista abierta. Las temáticas o las pláticas fueron efectuadas a los directivos de las organizaciones de manera directa e incluía temáticas sobre la creación del conocimiento, los modelos de gestión del conocimiento, la visión organizacional de los líderes para la apertura a la gestión del conocimiento. Como resultados principales de lo anterior se tiene que el poco interés que le propician a la gestión del conocimiento a través de un programa de gestión es casi nulo y parece que no tienen la intención de hacerlo, sin embargo, el conocimiento tácito y el tecnológico es adecuado ya que es el que les genera resultados más concretos. Por lo anterior, se plantea la siguiente pregunta de investigación:

¿Existe una influencia de los factores culturales en la generación de conocimiento de las organizaciones agrícolas del Valle del Yaqui?

Hipótesis.

Hi: Los factores culturales se relacionan e influyen significativamente en la generación de conocimiento de las organizaciones agrícolas del Valle del Yaqui.

\section{Hipótesis específicas:}

H1. La gestión del conocimiento y los factores culturales se relacionan significativamente en las organizaciones agrícolas del VY.

H2. Entre más factores culturales se incrementa la gestión del conocimiento en las organizaciones agrícolas del VY 
Objetivo.

Analizar la influencia de los factores culturales en la generación de conocimiento de las organizaciones agrícolas del Valle del Yaqui para la construcción de un modelo teórico que describa una realidad.

\section{Marco teórico}

\section{Perspectivas del conocimiento}

Hay quienes afirman que el conocimiento se encuentra en la mente, sin embargo, para Mercer (1997) puede ser compartido o llevar un flujo de comunicación, en el cual se lleve un proceso de aprendizaje, y menciona que agruparlo en dos mentes es mejor que tenerla en una, ya que para la construcción del mismo el resultado se vuelve más dinámico al compartir elementos como la cultura. Derivado de las líneas anteriores se puede llegar a la definición del conocimiento, misma que puede ser un conjunto de experiencias, valores e información dentro de un contexto el cual se puede utilizar para incorporar nuevas experiencias e información, y son las personas a través de su pensamiento quienes realizan esta incorporación, lo anterior fue expuesto por Davenport y Prusak (1998) mientras que para Nonaka y Takeouchi (1995) es un proceso humano dinámico de justificación de la creencia personal en busca de la verdad.

\section{Conocimiento tácito}

Es un tipo de conocimiento que todo ser humano posee, tal como lo menciona Busch (2008), es lo opuesto al conocimiento codificado o estructurado el cual cuenta con la característica de ser libre y encontrarse en cada individuo además de poseer un acceso restringido a otros individuos más. En términos generales se puede definir como las ideas y pensamientos que viven libremente y son entendidas de manera personal. Por otro lado, es esencial reconocer que descubrir en cada individuo este tipo de conocimiento para un investigador resulta en ocasiones un tanto cuanto complicado. Von Krogh, Ichijo y Nonaka (2001), comentan que una de las maneras más complicadas de transferir ese conocimiento es cuando se relaciona con tareas sumamente complejas como la interpretación de tendencias económicas, es por ello que el compartimiento del conocimiento tácito es un componente central de las organizaciones dedicadas a crear conocimiento.
Derivado de lo anterior, es importante describir algunos medios comunes para compartir el conocimiento tácito como: la observación directa que comprende darse cuenta de lo que hacen los individuos tanto como la relación maestro-discípulo, por otro lado, se encuentra la observación directa y descripción oral, misma que se apoya en relatos de otros individuos que han observado al trabajador, y por último aparece la imitación, lo que destaca es que los miembros tratan de adaptar lo que hacen los demás. Como ya se mencionó con anticipación que el conocimiento tácito es cada vez más complicado de interpretar y es difícil de reconocer es el conocimiento que a menudo conduce a avances significativos y del mismo modo genera más valor en la organización con un porcentaje sobresaliente contra el conocimiento explícito (Walkman, 2009).

\section{Conocimiento explícito}

Se le conoce como el causante de que exista la transferencia de conocimiento y se externalice, se expresa de manera formal y es visible tal como lo comentan Nonaka y Takeuchi (1995) puede comunicarse o difundirse con facilidad. En ese sentido Boisot (1998), comenta que lo rutinario que se presenta en el conocimiento tácito es totalmente distinto en el conocimiento explícito. Por lo anterior, cabe señalar que el conocimiento explícito es parte esencial de ese constructo llamado gestión del conocimiento. Debido a las articulaciones y que las organizaciones generan a manera de gestión con otras organizaciones es donde se aprecia de mejor forma la utilidad del desarrollo de este tipo de conocimiento. Por el análisis mencionado anteriormente, Ann y Souder (2015), comentan que la transferencia del conocimiento explícito es una función útil para la integración de los procesos o elementos de la misma organización y a su vez se puede incrementar y compartir con los mismos aliados organizacionales. Para las organizaciones que buscan la gestión del conocimiento es necesario propiciar la integración de los tipos de conocimiento según Spender (1996) una visión alternativa para la gestión del conocimiento propone una interacción entre tipos de conocimiento.

\section{Conocimiento cultural}

Este tipo de conocimiento es particular y su estudio es interesante en cuestión de los aspectos organizacionales.

FLORES-LÓPEZ, José Guadalupe, OCHOA-JIMÉNEZ, Sergio y TORRES-VÁSQUEZ, María del Carmen. El análisis de los elementos culturales como influencia de generación de conocimiento en las organizaciones agrícolas del Valle del Yaqui. Revista de Sociología Contemporánea. 2019 
Según Choo (1999) son las estructuras de conocimiento y de afecto que son utilizadas por los mismos miembros de la organización con el fin de poder explicar una realidad a través de opiniones y valores que otorgan un significado a la nueva información que ingresa o se construye a través de la combinación y transferencia del conocimiento.

De lo anterior hay diversas maneras de explicar cómo se identifica este tipo de conocimiento, Sackmann (1991) expone que existen cuatro formas de conocimiento cultural, se trata del conocimiento de diccionario que se trata para buscar las definiciones establecidas dentro de una organización para identificar el qué de cada situación, por otra parte se encuentra el conocimiento orientado y se atribuye a las prácticas de la organización haciendo alusión al cómo se realizan los procesos dentro de la empresa, en el siguiente se denomina conocimiento de recetas y se entiende como la forma en la cual se deberían resolver los problemas o situaciones y por último el conocimiento axiomático se refiere a identificar por qué dentro de la organización suceden las cosas.

\section{Cultura y su impacto en las organizaciones}

Es preciso señalar los orígenes de este concepto, la cultura como concepto de estudio tiene sus orígenes en los estudios antropológicos. Tal es el caso del Británico Edward Tylor, quien fue el primero en estudiarlo con profundidad. Por lo tanto, menciona que la cultura "es todo aquel complejo que incluye conocimiento, creencias, arte, moral, derecho, costumbres y cualquier otra capacidad y hábitos adquiridos por el hombre como miembro de la sociedad" (Tylor, 1871, p.1).

En relación a este último, surgieron diferentes posturas que trataron de explicar esta variable y han diferido entre sí. Para Merton, Weber, Durkheim y Marx citados por Alexander y Seidman (1942) el estudio de la cultura proveniente de lo social no con un enfoque antropológico como el caso de Tylor. En contraste, Parsons (1968) se enfoca a la cultura de acción establecida por el simbolismo que esta representa; por su parte Geertz (1989) realiza una conceptualización de la cultura a través del hombre y, finalmente Luhmann (1997) analiza una concepción histórica basada en los cambios a través del tiempo.
De igual manera, Geertz (1987), define la cultura como un patrón de concepciones heredadas. Por su parte, la cultura según Hosftede (1980 p. 43) "consiste en las reglas no escritas del juego social, es la programación colectiva de la mente que distingue a los miembros de un grupo o categoría de personas de otros". Mientras que la Real Academia Española (2001), se refiere a la cultura en general como el conjunto de formas $y$ costumbres de vida en un grupo social, un concepto similar es el de Díaz (2006), que muestra la cultura como las creencias, que se desarrollan o son descubiertas en grupos las cuales les permiten adaptarse al medio.

De lo mencionado, se puede compartir esas ideas siempre y cuando en punto particular se identifique también el liderazgo que ejerce el individuo en la organización, tal como lo explica Schein (1985), la cultura en las organizaciones por lo regular es creada por líderes que manejan artefactos y símbolos, éstos hacen que los empleados se vean influenciados en su comportamiento organizacional (ver Tabla 3).

\begin{tabular}{|r|l|l|}
\hline \multicolumn{1}{|c|}{ Año } & \multicolumn{1}{|c|}{ Autor(es) } & \multicolumn{1}{c|}{ Enfoque-Elemento } \\
\hline 1871 & Edward Tylor & Antropológico \\
\hline 1942 & $\begin{array}{l}\text { Merton, } \\
\text { Weber, } \\
\text { Durkheim y } \\
\text { Marx Citados } \\
\text { por Alexander } \\
\text { yeidman }\end{array}$ & Socialista \\
\hline 1968 & Parsons & Simbolismo \\
\hline 1980 & Hosftede & $\begin{array}{l}\text { Corporativa y de } \\
\text { grupos }\end{array}$ \\
\hline 1985 & Schein & $\begin{array}{l}\text { Organizacional y } \\
\text { Liderazgo }\end{array}$ \\
\hline 1987,1989 & Geertz & Estudio del hombre \\
\hline 1997 & Luhmann & $\begin{array}{l}\text { Concepción } \\
\text { Histórica }\end{array}$ \\
\hline & & \\
\hline
\end{tabular}

Tabla 3 El enfoque de la cultura desde sus inicios Fuente: elaboración propia

Existen diversas miradas que a lo largo de la historia han otorgado un sentido social desde la perspectiva cultura a la organización. Hay dentro de la literatura contemporánea los debates sociales que ofrecen una diversidad rica en cuanto a esas perspectivas que se comentan. El trabajo de Alexander y Seidman (1942) ofrece siete miradas distintas entre las cuales se destacan Funcionalista, Semiótica, Dramaturgia, Weberianismo, Durkheimiana, Marxismo y el Post-Estructuralismo. 
Es importante de manera general otorgar un repaso de cada una de las perspectivas que ofrecen Alexander y Seidman. La primera de ellas se le asume al problema de Merton y es enfocado al funcionalismo, la belleza de esta postura recae en la habilidad para entrelazar un énfasis cultural con un análisis de real acción social, es decir su carácter principal se basa en demostrar funcionalmente lo que realmente es probable y sugiere por lo cual Merton desarrolló un conjunto de elementos culturales que apoyan lo anterior. De la propuesta de la autonomía cultural la parte semiótica en resumen articula de una manera adecuada el conjunto de elementos culturales mediante niveles, por su pare la postura Dramaturgia articula de una manera propositiva la autonomía cultural del funcionalismo otorgando a cada individuo un rol especial.

Las aportaciones del Weberianismo y Durkheimiana se relacionan ampliamente por el componente cultural de la religión como elemento central en el cual la parte teológica juega un papel importante en las comunidades y socialmente es quién genera un poder $y$ encabezan un orden estructural. En el sentido apuesto anterior aparece el Marxismo y su forma autónoma de hacer las cosas de proponer una cultura basada en la vida económica y el PostEstructuralismo aparece como una crítica a las ideologías expuestas en las estructuras del Marxismo. Lo anterior, es un repaso general de cómo se han establecido culturalmente con relación a la sociedad, por ello es interesante el contraste con las perspectivas que se pueden visualizar específicamente en el ámbito organizacional. Para esto, Martin (1992), presenta las tres perspectivas organizacionales: la perspectiva de la integración (homogeneidad y armonía) la perspectiva de la diferenciación (separación y conflicto) y por último la perspectiva de Fragmentación (fluidez y multiplicidad).

Dentro de la primera perspectiva se trata que los individuos integren valores básicos, en la cual se busca que en toda la organización exista una consistencia y claridad. De la segunda se puede destacar los trabajos de las relaciones de poder, los conflictos organizacionales, las subculturas y las ambigüedades que relacionan a la separación de esos conflictos.
Y por último la tercera se refiere a que la organización inicie una fluidez en cuestión de las atenciones y las relaciones entre los manifestantes que buscan la multiplicidad de interpretaciones en consensos estables dentro de la organización.

Cabe señalar que las tres perspectivas se pueden interrelacionar con las formas expuestas a través de la historia de las cuales Alexander y Seidman hacen mención, el detalle está en identificar la postura que cada organización maneje internamente y con qué ideología opera su líder y distribuye hacia los demás miembros de la organización. Las perspectivas anteriores han demarcado una serie de expresiones que describen a la cultura en las organizaciones como una variable que se puede estudiar desde diferente apreciación según el enfoque con que se perciba (ver Tabla 4).

\begin{tabular}{|c|c|c|c|}
\hline Expresión & Corporativa & $\begin{array}{c}\text { Perspectiva cultural } \\
\text { Organizacional- } \\
\text { Determinista }\end{array}$ & $\begin{array}{l}\text { Organizacional- } \\
\text { Simbólica }\end{array}$ \\
\hline Objetivo & $\begin{array}{l}\text { Incremento en la } \\
\text { utilidad }\end{array}$ & $\begin{array}{l}\text { Comprensión del } \\
\text { Comportamiento }\end{array}$ & $\begin{array}{l}\text { Comprensión del } \\
\text { sentido de la } \\
\text { acción }\end{array}$ \\
\hline Enfoque & $\begin{array}{l}\text { Variable } \\
\text { Estratégica } \\
\end{array}$ & Factores & \\
\hline Establecimiento & $\begin{array}{l}\text { Cuadros } \\
\text { Directivos } \\
\end{array}$ & \multicolumn{2}{|c|}{ Miembros de la Organización } \\
\hline Intención & Cultura Meta & Conocimiento de la C & ultura \\
\hline $\begin{array}{l}\text { Orientación } \\
\text { Metodológica }\end{array}$ & \multicolumn{2}{|c|}{ Cuantitativa en base a cuestionarios } & $\begin{array}{l}\text { Cualitativa, con } \\
\text { base a entrevistas }\end{array}$ \\
\hline $\begin{array}{ll}\text { Elementos } & \mathrm{de} \\
\text { Observación }\end{array}$ & \multicolumn{2}{|l|}{ Comportamientos } & $\begin{array}{l}\text { Rituales, valores, } \\
\text { actitudes y mitos }\end{array}$ \\
\hline $\begin{array}{l}\text { Naturaleza de la } \\
\text { Acción }\end{array}$ & Voluntarismo & Determinismo & Reproducción \\
\hline $\begin{array}{l}\text { Relaciones entre } \\
\text { individuos }\end{array}$ & Armónicas & $\begin{array}{l}\text { Relativamente } \\
\text { armónicas }\end{array}$ & $\begin{array}{l}\text { Contradictorias y } \\
\text { conflictivas }\end{array}$ \\
\hline Resultados & $\begin{array}{ll}\begin{array}{l}\text { Diversas } \\
\text { exterior, }\end{array} & \text { al } \\
\text { homogénea } & \text { al } \\
\text { interior. } & \\
\end{array}$ & $\begin{array}{l}\text { Isomorfismo } \\
\text { estructural }\end{array}$ & Diversidad \\
\hline $\begin{array}{l}\text { Disciplinas } \\
\text { Principales }\end{array}$ & $\begin{array}{l}\text { Administración, } \\
\text { psicología } \\
\text { organizacional. }\end{array}$ & $\begin{array}{l}\text { Administración, } \\
\text { Teoría de la } \\
\text { organización }\end{array}$ & $\begin{array}{l}\text { Antropología, } \\
\text { estudios } \\
\text { organizacionales }\end{array}$ \\
\hline $\begin{array}{l}\text { Horizonte de } \\
\text { Cambio }\end{array}$ & Corto Plazo & Largo Plazo & \\
\hline $\begin{array}{l}\text { Organizaciones } \\
\text { Estudiadas }\end{array}$ & \multicolumn{2}{|c|}{ Principalmente Empresas } & $\begin{array}{l}\text { Organizaciones } \\
\text { Diversas }\end{array}$ \\
\hline $\begin{array}{l}\text { Nivel de } \\
\text { Comparación }\end{array}$ & \multicolumn{2}{|c|}{$\begin{array}{l}\text { Muy alto entre organizaciones y a nivel } \\
\text { mundial }\end{array}$} & Mediano \\
\hline $\begin{array}{l}\text { Autores } \\
\text { representativos }\end{array}$ & Edgar Schein & Geert Hofstede & Mats Alvesson \\
\hline
\end{tabular}

Tabla 4 Expresiones más comunes de la Cultura en las Organizaciones

Fuente: elaboración propia. Adaptado de Rendón (2007)

Al final, se puede considerar que las diferentes perspectivas engloban aspectos que se asemejan para las condiciones del estudio de la cultura en las organizaciones. Por ejemplo, aspectos como la unidad, ritos, comportamiento y lenguaje hace que complementen aspectos interesantes para el estudio de la cultura tanto de manera individual como colectiva (Rendón, 2007). 


\section{Método}

Para cumplir con el propósito de esta investigación se eligieron los participantes de la misma. En ese sentido, se dio a la tarea de seleccionar productores agrícolas que desarrollan su actividad de siembra y cosecha con una diversidad de cultivos como: trigo, maíz, cártamo, higuerilla, tomate, chile, papa, espárragos y algodón. Se obtuvieron 90 encuestas aplicadas. De las personas que respondieron el instrumento el $94 \%$ de ellos son del sexo masculino y tan solo el $6 \%$ pertenecen al sexo femenino, por lo que se entiende que en mayor parte el sector agrícola es manejado por hombres y se caracterizan en diversos puestos de trabajo (ver Tabla 5).

\begin{tabular}{|l|r|r|}
\hline \multicolumn{1}{|c|}{ Puesto } & Frecuencia & Porcentaje \\
\hline Productor (Agricultor) & 57 & 64 \\
\hline Administradores & 28 & 30 \\
\hline Técnico-Supervisores & 5 & 6 \\
\hline Total & 90 & 100 \\
\hline
\end{tabular}

Tabla 5 Número de personas encuestadas por puestos Fuente: elaboración propia

De igual manera se aplicaron siete entrevistas a profundidad constituidas de la siguiente manera: tres personas físicas, cuatro personas morales, con características de: baja y alta superficie de siembra, y miembros activos de asociaciones de productores agrícolas (ver Tabla 6).

\begin{tabular}{|l|r|}
\multicolumn{1}{|c|}{ Características } & 3 \\
\hline Personas físicas & 4 \\
\hline Personas morales & 4 \\
\hline $\begin{array}{l}\text { Socio de unión de crédito y/ } \\
\text { organismos de productores. }\end{array}$ & 5 \\
\hline Cultivo grano & 2 \\
\hline Cultivo hortaliza y/ otro & 5 \\
\hline Superficie ejidal & 3 \\
\hline Superficie baja & 4 \\
\hline Superficie alta & \\
\hline
\end{tabular}

Tabla 6 Características de los entrevistados $(\mathrm{N}=7)$ Fuente: elaboración propia

Se determinó elaborar un cuestionario de escala tipo Likert constituido por cinco puntos de respuesta con un total de 28 ítems para las dos variables de estudio (ver Tabla 7).

\begin{tabular}{|l|l|l|}
\hline \multicolumn{1}{c}{ Variable Ítems } & \multicolumn{2}{c|}{ Autores } \\
\hline $\begin{array}{l}\text { Gestión del } \\
\text { Conocimiento }\end{array}$ & $1-14$ & $\begin{array}{l}\text { (Jones, 2009; Bueno, } \\
\text { 1999; Nonaka \& } \\
\text { Takeuchi, 1995) }\end{array}$ \\
\hline $\begin{array}{l}\text { Cultura } \\
\text { Organizacional }\end{array}$ & $15-28$ & $\begin{array}{l}\text { (Jones, 2009; Schein, } \\
1985 ; \text { y Hosftede, 1980) }\end{array}$ \\
\hline
\end{tabular}

Tabla 7 Estructura del instrumento de recolección Fuente: elaboración propia

\section{Resultados}

Se desarrollaron la prueba de correlación y regresión lineal Pearson con las cuales se aceptan las hipótesis específicas 1 y 2 planteadas en el apartado número uno del proyecto:

- H1. La gestión del conocimiento y los factores culturales se relacionan significativamente.

- H2. Entre más factores culturales se incrementa la gestión del conocimiento en las organizaciones agrícolas del VY.

En ese sentido, como resultado de las pruebas estadísticas efectuadas se tiene que la cultura organizacional y la gestión del conocimiento se relacionan entre sí con una eficiencia positiva mayor a uno, misma que representa un porcentaje medio del $33 \%$ en un nivel de significancia del .01 el cual indica el riesgo a equivocarse considerando la aceptación del supuesto establecido y rechazar la hipótesis nula (ver Tabla 8). Es por ello que, la cultura de las organizaciones agrícolas mantiene un nivel de aceptación al utilizar sus costumbres y tradiciones para generar actividades de gestión del conocimiento según los datos presentados en los ítems CUL2 y CUL4 en los que ambos presentan una media superior al 3.2 respectivamente.

\begin{tabular}{|l|c|c|r|r|r|}
\hline \multicolumn{1}{|c|}{ Variable } & $\mathbf{N}$ & $\mathbf{M}(\%)$ & SD (\%) & $\mathbf{1}$ & $\mathbf{2}$ \\
\hline $\begin{array}{l}\text { Gestión del } \\
\text { conocimiento }\end{array}$ & 90 & 3.59 & .85 & - & - \\
\hline $\begin{array}{l}\text { Cultura } \\
\text { organizacional }\end{array}$ & 90 & 3.07 & .94 & .331 & - \\
\hline Nota: Significativa al .01 (2 colas) \\
\hline
\end{tabular}

Tabla 8 Correlación entre GC y CUL

Fuente: elaboración propia 
Dentro del marco mencionado con anterioridad, se establece que el conocimiento que se genera y se transmite en el valle del yaqui es por tradición y, en general se realizan las mismas actividades año con año; tal como lo menciona el entrevistado 1 "hay comentarios que me han hecho a mí: es que así lo hacía mi tío, mi tía, mi abuela, generación tras generación lo están haciendo" (E1) y ese conocimiento generado si les da resultado no lo cambiarán como lo menciona el entrevistado 3 "esa es ya como tradición de nosotros, ser los primeros, sembrar y lo seguimos haciendo porque así nos gusta, nos da resultados, hemos encontrado menos plagas (E3) pero en gran parte se siembra y se hacen tareas por ideologías o jerarquías como lo hace notar el entrevistado 2 "por la traición más por nada...Mi papá es el que le gusta el trigo y a mí no me gusta el trigo a mí me gusta el maíz" (E2) y esas actividades seguirán así aunque les genere más costos menciona el entrevistado 6 "al productor del valle del yaqui en general no le gusta sembrar otra cosa más que trigo, y cuando le preguntas por qué no cambias por qué no diversificas, te responden que porque tienen mucha maquinaria" (E6).

Por lo tanto, derivado de la relación entre las variables se manifiesta una influencia en la cual la cultura organizacional influye directamente en la generación del conocimiento según el cálculo de regresión lineal efectuado en el cual se observa que la cultura predice en un $9 \%$ el conocimiento que se genera en el valle del yaqui (ver Tabla 9).

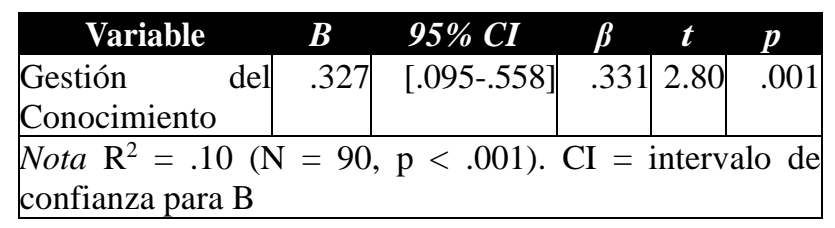

Tabla 9 Regresión Lineal entre CUL y GC Fuente: elaboración propia

Las evidencias anteriores, definen la cultura organizacional no juega un papel importante dentro de la generación del conocimiento ya que no se aprecia un impacto que llegue a determinar un cambio en la creación, aplicación y transferencia del conocimiento en el valle del yaqui. Si bien, lo anterior resulta significativo, para muestras no tan extensas el nivel de influencia no se considera alto.
Por lo tanto, se determina que en el valle del yaqui los productores al incrementar sus actividades culturales no predicen una generación de conocimiento, tal como lo explica el entrevistado 1 "la cultura no refleja el hecho de que busque uno u otro cultivo para vivir mejor, son conformistas en lo que tienen" (E1). En ese sentido, lo que los hace fuerte a las organizaciones de productores es la estructura que les brindan las propias instituciones como bien menciona el entrevistado 1 "si hablamos en forma de organización, en el valle del yaqui encontramos su fortaleza porque son instituciones que son propiamente antiguas $y$ con fuerza, como las uniones, las asociaciones y estas funcionan como tal (E1) y propiamente porque les brinda la seguridad financiera, que ya lo hacen por costumbre y una necesidad para realizar la actividad no para generar conocimiento ni nuevas formas de producción "la financiera rural me da el crédito para los cultivos" menciona el entrevistado 2 y concluye el entrevistado 1 "toman como una cultura el financiarse" (E1).

\section{Conclusiones}

Como reflexiones finales, el objetivo del proyecto se ha cumplido de manera satisfactoria y es importante mencionar que los resultados muestran la relación tan directa que existe entre los factores culturales como las creencias y tradiciones con la generación de conocimiento en el Valle del Yaqui, sin embargo, ellas mismas fungen como barreras para la creación de nuevo conocimiento. De igual manera, se determina una primera fase de estudio de estas temáticas en el sector, con el fin de establecer mejoras y construir modelos que aporten nuevos conocimientos y se pueda explicar la realidad cultural en la que se desarrolla el Valle del Yaqui. Ampliar con estudios de modernidad y tradición sería una buena oportunidad para crecer en el análisis de las comunidades agrícolas, así como el estudio de la familia y su cultura. Por lo tanto, sería interesante la comparación de un estudio similar en otro contexto agrícola y llegar a resultados más cercanos para explicar el fenómeno en toda su magnitud. 


\section{Referencias}

Appendini, K. A. D., Martínez, M. P., Rendón Gan, M. T., \& Salles, V. (1983). El campesinado en México: dos perspectivas de análisis.

Arnold-Cathalifaud, M., Thumala, D., \& Urquiza, A. (2007). Colaboración, cultura y desarrollo: entre el individualismo y la solidaridad organizada. Revista Mad. Revista del Magíster en Análisis Sistémico Aplicado a la Sociedad, (2), 15.

Camiro Pérez, M. A., Altamirano Cárdenas, R., \& Rojas Herrera, J. J. (2009). Retos del crédito agrícola: Estudio de caso de la intermediación financiera en el sur de Sonora, México. Región y sociedad, 21(46), 53-78.

Cerutti y Lorenzana (2009 (2004), Subrayando "cultura" en "agricultura" http://www.fao.org/docrep/018/i2940s/i2940s0 9.pdfFayol, H., \& Taylor, F. W. (1987). Administración industrial y general. Barcelona: Orbis.

http://maryperez.galeon.com/admonindustrial.p df

Choo, C. W., \& Díaz, D. R. (1999). La organización inteligente: el empleo de la información para dar significado, crear conocimiento y tomar decisiones. México DF: Oxford University Press.

Díaz Sánchez, J. (2006). Identidad, adolescencia y cultura. Jóvenes secundarios en un contexto regional. Revista mexicana de investigación educativa, 11(29), 431-457.

Explorando México (2012). La Agricultura en México, recuperado de https://www.explorandomexico.com.mx/aboutmexico/8/407 el 06 de septiembre de 2016

FAO, L. F. (2014). en México; más de 60 años de cooperación 1945-2009. Consultado en http://coin. fao. org/cms/world/mexico/InformaccionSobreElPai s/sector_agroalimenta rio. html el, 25.

Fiske, S. T., \& Taylor, S. E. (2013). Social cognition: From brains to culture. Sage.
Gálvez, M. P. G. (2009). Factores facilitadores para las prácticas de Gestión del Conocimiento y de Inteligencia Competitiva. Cuadernos de inteligencia competitiva, vigilancia estratégica, científica y tecnológica (QUIC\&VECT), 2(1), 45-59.

García Cabrero, B., \& Pineda Ortega, V. J. (2010). La construcción de conocimiento en foros virtuales de discusión entre pares. Revista mexicana de investigación educativa, 15(44), 85-111.

Geertz, C. (1987). Descripción densa: hacia una teoría interpretativa de la cultura. La interpretación de las culturas, 19-40.

Geertz, C. (1989). El impacto del concepto de cultura en el concepto de hombre. La interpretación de las culturas. Barcelona: Gedisa, 43-59.

Giménez, G. (1996). Territorio y cultura. Estudios sobre las culturas contemporáneas, 2(4), 9-30.

Gómez, D. R. (2006). Modelos para la creación y gestión del conocimiento: una aproximación teórica. Educar, 37, 25-39.

http://www.redinnovagro.in/casosexito/48sonor auvademesa.pdf

INEGI

http://www3.inegi.org.mx/sistemas/Movil/Mexi coCifras/mexicoCifras.aspx?em $=26018 \& \mathrm{i}=\mathrm{e}$

INEGI, C. E. (2009). Instituto Nacional de Estadística y Geografía. Censo Económico.

INEGI, I. (2005). Conteo de población y vivienda 2005. Indicadores del censo general de Población y vivienda, Ed. INEGI, México.

INEGI, M. (2010). México en cifras. Recuperado el, 11 de septiembre de 2011 http://www3.inegi.org.mx/sistemas/Movil/Mexi coCifras/mexicoCifras.aspx $? \mathrm{em}=26018 \& \mathrm{i}=\mathrm{e}$

Leal Soto, S. D., Valenzuela Quintanar, A. I., Gutiérrez Coronado, M. D. L., Bermúdez Almada, M. D. C., García Hernández, J., Aldana Madrid, M. L., ... \& Palma Durán, S. A. (2014). Residuos de plaguicidas organoclorados en suelos agrícolas. Terra latinoamericana, 32(1), $1-11$. 
Liquori, C. L., Ricker, K., Moseley, M. L., Jacobsen, J. F., Kress, W., Naylor, S. L., ... \& Ranum, L. P. (2001). Myotonic dystrophy type 2 caused by a CCTG expansion in intron 1 of ZNF9. Science, 293(5531), 864-867.

López, J. G. F., \& Jiménez, S. O. Los modelos de gestión del conocimiento y su relación con la cultura organizacional: Una revisión teórica (The models of knowledge management and its relation to the organizational culture: a theoretical review).

López, J. G. F., \& Jiménez, S. O. Los modelos de gestión del conocimiento y su relación con la cultura organizacional: Una revisión teórica (The models of knowledge management and its relation to the organizational culture: a theoretical review).

Martínez-Hernáez, A. (2011). El dibujante de limites: Franz Boas y la (im) posibilidad del concepto de cultura en antropologia. História, Ciências, Saúde-Manguinhos, 18(3), 861-876.

Miranda, M. A. A., Rivera, É. S. R. S., \& González, T. B. B. (2011). El clima como manifestación objetiva de la cultura organizacional. Revista Ciencias Estratégicas, 19(25), 109-

Morales Martinez, m. A., Walsh Rodriguez, C., Ruelas Monjardin, 1. C., Weissling, b., Sieron, k., \& Ochoa Martinez, c. A. (2016). Afectaciones por posible asociación de eventos hidrometeorológicos en los municipios de Calcahualco y Coscomatepec Veracruz.

Moreno, A., Del Villar, F., García-González, L., García-Calvo, T., \& Moreno, M. P. (2012). Propiedades psicométricas de un cuestionario para la evaluación del conocimiento procedimental en voleibol (CCPV). Psychometric properties of Procedural Knowledge Questionnaire in Volleyball (PKQV). RICYDE. Revista Internacional de Ciencias del Deporte. doi: 10.5232/ricyde, 9(31), 38-47.
Moreno-villa, e. D., Aldana-madrid, M. L., Silveira-Gramont, m. I., Rodríguez-Olibarría, g., Valenzuela-Quintanar, a. I., \& MezaMontenegro, m. (2012). Análisis de piretroides en suelo y agua de zonas agrícolas y urbanas de los valles del Yaqui y Mayo. Revista internacional de contaminación ambiental, 28(4), 303-310.

Naylor, R. L., Falcon, W. P., \& Puente Gonzalez, A. (2001). Policy reforms and Mexican agriculture: views from the Yaqui Valley (pp. 26-pages). CIMMYT.

Portal, M. A., Álvarez, L., Camarena, M., Giglia, Á., Gomezcésar, I., Mejorada, C. S., ... \& Negrete, M. P. (2012). Ciudad global, procesos locales: conflictos urbanos y estrategias socioculturales en la construcción de sentido de pertenencia y del territorio en la Ciudad de México. Proyecto de investigación, CONACYT, México.

Pugna, I. B., \& Boldeanu, D. M. (2014). Factors affecting establishment of an institutional knowledge management culture-a study of organizational vision. Accounting and Management Information Systems, 13(3), 559.

Ramírez Díaz, J. M. (2008). Análisis foliar para el manejo nutrimental de huertas de naranja Valencia en el Valle del Yaqui, Sonora. In XI Congreso Internacional de Ciencias Agrícolas. Univ. Autónoma de BC Mexicali, BC Octubre (Vol. 23).

Ranucci, R. A., \& Souder, D. (2015). Facilitating tacit knowledge transfer: routine compatibility, trustworthiness, and integration in $\mathrm{M} \&$ As. Journal of Knowledge Management, 19(2), 257276.

Reyes Meleán, C. F. (2005). Análisis de la relación entre la ingeniería del conocimiento y la gestión del conocimiento en base al modelo de Nonaka y Takeuchi. Reyes Meleán, Christian Fernando." Anáisis de la relación entre la ingeniería del conocimiento y la gestión del conocimiento en base al modelo de Nonaka y Takeuchi". Intangible Capital, julio-septiembre de 2005, vol. 1, núm. 9, 15 p. 
Reyes Meleán, C. F. (2005). Análisis de la relación entre la ingeniería del conocimiento y la gestión del conocimiento en base al modelo de Nonaka y Takeuchi. Reyes Meleán, Christian Fernando." Anáisis de la relación entre la ingeniería del conocimiento y la gestión del conocimiento en base al modelo de Nonaka y Takeuchi". Intangible Capital, julio-septiembre de 2005, vol. 1, núm. 9, 15 p.

SAGARPA, M. (2009). Programa de Producción Sustentable de Insumos para Bioenergéticos y de Desarrollo Cientifico y Tecnológico. G. Secretarıa de Agricultura, Desarrollo Rural, Pesca y Alimentación, (ed.).

SAGARPA, S. (2007). Secretaria de agricultura, ganadería, desarrollo rural, pesca y alimentación; servicio de información agroalimentaria y pesquera.

Servicios de Información Agroalimentaria y Pesquera (2012). Datos Estadísticos del año 2012 Consultado el 8 de agosto de 2014 Secretaría de Economía (2012) www.economia.gob.mx

SIAP, S. (2014). Servicio de información agroalimentaria y pesquera.

Silos José, S., \& Donald, F. (1964). Economía Agrícola del Noroeste. La agricultura en el sistema de pequeña propiedad.

Sparrow, P. R. (2002). Gesti $\tilde{A}^{3} n$ del conocimiento, aprendizaje organizacional y psicolog $\tilde{A} a \quad$ cognitiva: desentra $\tilde{A} \pm$ ando importantes competencias individuales y organizacionales. Journal of Work and Organizational Psychology, 18(2-3), 131-156.

Velasco Magallanes, J. E., Pérez López, D. D. J., Rajaram Devi, S., Balbuena Melgarejo, A., Albarrán Mucientes, M., \& González Huerta, A. (2012). Análisis de 20 genotipos de trigo harinero en el Valle del Yaqui, Sonora. Revista mexicana de ciencias agrícolas, 3(8), 15211534. 